\title{
Peter Hänni: Rosas Blut (2)
}

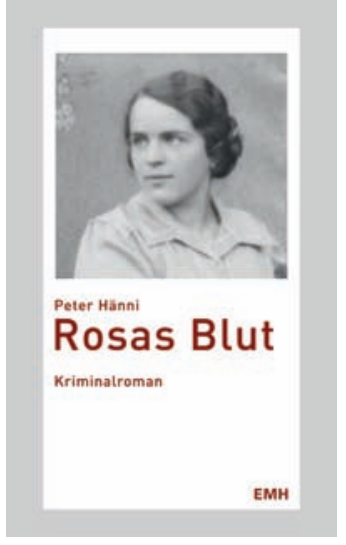

\section{Peter Hänni}

\section{Rosas Blut}

EMH Schweizerischer Ärzteverlag

Kriminalroman

2008. 192 Seiten. Broschiert.

Fr. $21.50 / € 14.80$

exkl. Versand

ISBN 978-3-0375-033-6

Was würde ihn in Montella erwarten? Als kleiner Junge war er das letzte Mal dort gewesen, vor vierunddreißig Jahren. Viel mehr als einige bruchstückhafte Erinnerungen waren nach der langen Zeit nicht in seinem Bewusstsein hängengeblieben. An das Haus seiner inzwischen verstorbenen Großmutter zum Beispiel, an enge, nach Urin stinkende, beidseitig von uralten Steinhäusern gesäumte Gassen, über die zahllose mit Wäschestücken behängte Leinen gespannt waren. Oder an die paar zerzausten Hühner, die im Hinterhof des Hauses herumstelzten, bis sie das Schicksal in Form von Großmutters Axt ereilte.

Ihn hatte man zusammen mit seinen Eltern und seiner Schwester im Schlafzimmer der alten Frau einquartiert. $\mathrm{Zu}$ viert hatten sie sich in ihr Bett pferchen müssen, und manchmal, wenn er in den heißen Sommernächten keinen Schlaf hatte finden können, hatte er durch den Vorhang hindurch seine Großmutter auf dem winzigen Balkon im Schaukelstuhl sitzen sehen, bewaffnet mit einer alten Flinte, die sie sich quer über den Schoß gelegt hatte. «Um die Hühner vor dem Fuchs zu schützen», hatte sie ihm damals erklärt.

Er erinnerte sich, dass viele Leute in Großmutters Haus ein- und ausgegangen waren. Menschen, die gekommen waren, um die aus der Schweiz angereiste Familie seines Vaters zu begrüßen. Und von all diesen zwar liebenswerten, ihm aber doch fremden Personen musste er sich fortwährend knuddeln, in die Wangen kneifen und abküssen lassen. Nicht wenige von ihnen Männer und Frauen - hatten kratzige Stoppelbärte, fauligen Mundgeruch oder fehlende Zähne. Oder alles zusammen. Einige der Männer stanken nach abgestandenem Zigarettenrauch, und die überwiegend älteren Frauen waren in dunkelstes, bedrückendes Schwarz gekleidet. Nach Erduldung der ihm aufgezwungenen Zärtlichkeiten hatte er sich immer so schnell wie möglich zum Waschbecken davongeschlichen, um sich heimlich das Gesicht zu waschen. Die Zuneigung dieser Leute hatte ihn schier erdrückt. «Mein Gott, wie ich das gehasst habe!», flüsterte er mit einem leisen Seufzer.

In den letzten Jahren hatte er zu seinen italienischen Verwandten kaum Kontakt gehabt. Nur mit Carlo und dessen Familie hatte er sich selten, aber regelmäßig getroffen - zumindest an Ostern, zu einem gemeinsamen Mittagessen. Carlo hatte er gemocht. Ein humorvoller und großzügiger Mann, der viel und gerne gearbeitet hatte, obschon seine Tätigkeit in der Papierfabrik wahrscheinlich alles andere als ein Zuckerschlecken war. Mit ihm hätte er gerne ein wenig mehr Kontakt gehabt. Auch mit Francesco, seinem Cousin, hatte er sich stets gut verstanden. Von Tante Laura hingegen hielt er nicht viel. Sie war schuld, dass ihre Familien trotz geographischer Nähe immer ein Stück weit auf Distanz geblieben waren. Laura hatte seine Mutter nie richtig akzeptiert, weil sie Schweizerin gewesen war. Und sie hatte sie dies auch immer spüren lassen. Später war dann wohl noch eine gewisse Eifersucht dazugekommen, weil aus ihm, dem Sohn dieser Schweizerin, ein angesehener Arzt geworden war, während sich Francesco mit mäßigem Erfolg im Gastgewerbe durchzuschlagen versuchte.

Wie auch immer! Der Gedanke, dass er in Montella zahlreiche seiner ihm kaum bekannten Verwandten antreffen würde, behagte ihm nicht sonderlich. Er verzog leicht das Gesicht und rieb sich die Magengegend, wo er einen leichten Schmerz verspürte. Kurz darauf fiel er in einen oberflächlichen, unruhigen Schlaf.

Eine säuselnde, etwas undeutliche weibliche Stimme holte ihn wieder aus seinem Dämmerzustand. Über den Bordlautsprecher wurden die Passagiere informiert, dass in Kürze der Lande-

\section{Rosas Blut - ein Kriminalroman bei EMH}

Mit «Rosas Blut» von Peter Hänni erweitert der Schweizerische Ärzteverlag EMH sein Buchprogramm um die Sparte Kriminalroman. Leserinnen und Leser der SÄZ können sich von den Qualitäten des Romans anhand einer Leseprobe in vier Teilen überzeugen - und sie haben die Möglichkeit, das Buch direkt beim Verlag zu bestellen: online unter www.emh.ch/books oder über Tel. 06146785 75. Ein Artikel von Erhard Taverna zu Autor und Werk ist in der SÄZ Nr. 40/2008 erschienen. anflug beginnen werde. Sie wurden gebeten, die Sitze aufzurichten und sich anzuschnallen. Luca stellte fest, dass sein Vater ohne Verzögerung auf die Anweisungen der Lautsprecherstimme reagierte. Offenbar hatte auch er nicht sehr tief geschlafen.

Die Landung ließen beide in angespanntem Schweigen über sich ergehen.

Nachdem sie ihr Gepäck vom Laufband genommen und die Kontrollen passiert hatten, betraten sie die Ankunftshalle des Flughafens Capodichino. Die Uhr zeigte siebzehn Uhr fünfunddreißig. Suchend ließen sie ihre Blicke über die Menschenmenge schweifen, bis Luca einen grauhaarigen, ungefähr fünfzig- bis sechzigjährigen Mann entdeckte, der ihnen lebhaft zuwinkte. «Schau mal Pa, dort drüben!»

Salvatore erkannte ihn sofort und winkte zurück: «Das ist Aurelio!»

Der nicht sehr große, aber bullige Mann tankte sich problemlos durch die dichtgedrängte Menge und kam rasch auf sie zu. Nach einer kurzen und herzlichen Begrüßung forderte Aurelio die beiden auf, sich zu beeilen, da er möglichst noch vor dem Abendverkehr aus Neapel herauskommen wollte. Vom Flughafen Capodichino bis nach Montella musste man normalerweise mit einer Fahrzeit von eineinhalb Stunden rechnen, bei starkem Verkehr konnte die Reise in das neunzig Kilometer östlich von Neapel gelegene Dorf auch gut eine Stunde länger dauern. Aurelio führte die beiden Ankömmlinge zügigen Schrittes ins Flughafen-Parking, wo er neben einem blitzsauberen silbergrauen Alfa Romeo stehen blieb. «Da wären wir!», verkündete er und zeigte mit unverhohlenem Stolz auf den Wagen.

Die anschließende Fahrt nach Montella empfand Luca anstrengender als den ganzen bisherigen Tag. Er saß hinten im Wagen, der von Aurelio auf eine Art und Weise gelenkt wurde, die in ihm eine Mischung aus Angst, Übelkeit und Belustigung hervorrief. Im zunächst zähflüssigen Verkehr fluchte, hupte und gestikulierte Aurelio immerzu, während er den Alfa mit heftigen Beschleunigungs- und brüsken Bremsmanövern bocksprungartig vorantrieb. Am schlimmsten war jedoch die Fahrt auf der Landstraße, kurz vor Montella. Auf dieser Strecke fürchtete Luca 
zeitweilig um sein Leben, denn Aurelio fuhr nicht nur schnell, sondern vollführte wiederholt riskante Überholmanöver. Darüber hinaus schien er noch nie etwas von Sicherheitsabständen gehört zu haben. Und während er so dahinraste und sich durch den Verkehr rüpelte, hatte er auch einiges zu erzählen. Er unterhielt sich zunächst mit Salvatore, der neben ihm auf dem Beifahrersitz saß. Später richtete er das Wort auch an Luca, den Blickkontakt zu ihm über den Rückspiegel suchend.

«Und du, Luca? Oder muss ich dich jetzt ¿Dottore nennen? Es ist lange her, seit wir uns das letzte Mal gesehen haben. Wahrscheinlich über dreißig Jahre. Du warst damals noch ein kleiner Knirps. Erinnerst du dich?»

«Eigentlich kaum!», antwortete Luca reserviert und kam sich vor wie sein wortkarger Vater. Aber angesichts von Aurelios Fahrweise verspürte er nicht die geringste Lust, das Gespräch weiter in Gang zu halten. Der Mann sollte sich gefälligst auf die Straße konzentrieren!

Obschon, eigentlich konnte er sich noch ganz gut an den damals stets gut gelaunten jungen Mann mit dem langen Kleinfingernagel erinnern. Aurelio hatte ihn und seine kleine Schwester jeweils nach dem Abendessen in eine kleine Gelateria entführt und ihnen ein Eis spendiert. Wegen dieser abendlichen Ausflüge - und wegen des langen Fingernagels - hatte er bei Luca einen die Jahrzehnte überdauernden Eindruck hinterlassen. Mittlerweile war Aurelio um einiges schwerer, seine Haare grauer und sein Fingernagel wesentlich kürzer geworden.

Was Carlos Tod anbelangte, wusste Aurelio nichts Neues zu berichten. Nur dass die Abdankungsfeier morgen um elf Uhr in der Chiesa Santa Lucia stattfinden werde. Tante Giuseppina und Onkel Alfredo seien schon am Nachmittag eingetroffen und würden bei ihm und seiner Frau logieren. Er entschuldigte sich dafür, dass er sie, Salvatore und Luca, nicht auch bei sich beherbergen konnte, bot ihnen jedoch an, sie zu seiner jüngsten Schwester zu bringen. Maria Barbone und ihr Ehemann Cesare würden sich freuen, den beiden für ein paar Tage ihre Gastfreundschaft anbieten zu dürfen. Eigentlich hätte Luca ein Hotel vorgezogen, aber sein Vater nahm das Angebot sofort an, ohne ihn zu fragen.

In der Zwischenzeit hatte es eingedunkelt und angefangen zu regnen. Kurz nach sieben erreichten sie endlich Montella. Ein kleines, am Fuße des Monte Sassetano gelegenes Dorf, das sich über eine weite, zum Parco Regionale dei Monti Picentin gehörende Hochebene erstreckte, die wiederum Teil der Provinz Avellino war. Von seinem Vate wusste Luca, dass ungefähr achttausend Montellesi dort lebten, in einem sauberen Klima mit milden Wintern und nicht allzu schwülen Sommern.
Sie bogen in die im südlichen Teil des Dorfes gelegene Via Fondana ein, und nach wenigen Metern stoppte Aurelio den Wagen, unmittelbar vor dem Ristorante al Barbiere.

«Endstation!», verkündete er, stellte den Motor ab, stieg aus und zündete sich eine Zigarette an. Nach Salvatore schälte sich auch Luca aus dem Wagen, froh darüber, heil am Ziel angekommen zu sein. Er streckte sich und atmete die angenehm kühle Herbstluft tief ein.

«Die Barbones wohnen hier im zweiten Stock», erklärte Aurelio mit der Zigarette im Mundwinkel, während er den Kofferraum öffnete und die Gepäckstücke heraushievte. «Das Restaurant wird von Maria geführt. Cesare hat sich in einem Teil des Lokals einen kleinen Coiffeursalon eingerichtet. Man kann sich hier also nicht nur kulinarisch verwöhnen, sondern auch die Haare schneiden lassen! Aber heute und morgen ist natürlich geschlossen.» Er zog noch ein paar Mal kräftig an der Zigarette, dann schnippte er die Kippe auf die Straße und forderte die beiden auf, ihm zu folgen. Er begab sich zum Hauseingang, wo er mehrmals auf den einzigen Klingelknopf drückte.

Carlos Leichnam war im geräumigen Wohnzimmer aufgebahrt. Man hatte den offenen Sarg vor der Fensterfront platziert, die mit dicken, schweren Stoffvorhängen verkleidet war, so dass auch am hellen Tag kein Lichtstrahl den Weg ins Zimmer finden konnte. In dem von Kerzen schwach erleuchteten Raum waren einige Möbel zur Seite geräumt worden, dafür standen mehrere zusätzliche Stühle herum. Um den dunkelbraunen Sarg hatte man Trauergebinde und Heiligenbildnisse drapiert, von denen das zeitweilig in der Zugluft flackernde Kerzenlicht reflektiert wurde.

Im Lauf des Nachmittags und am frühen Abend fanden zahlreiche Freunde und Bekannte den Weg in die Wohnung des Verstorbenen, um ihm die letzte Ehre zu erweisen und den Hinterbliebenen ihre Anteilnahme auszudrücken. Es war ein ständiges Kommen und Gehen, und der Raum wurde immer wieder von leisem Schluchzen und Wimmern, bisweilen auch von ungehemmtem Wehklagen erfüllt - vor allem, wenn die näheren Verwandten des Verstorbenen sich darin aufhielten. Gesprochen wurde kaum, nur selten war ein leises Flüstern zu hören. Aus den Nebenräumen hingegen, wo sich die Besucher vor und nach dem Aufenthalt im Aufbahrungszimmer versammelten, vernahm man ein ständiges Gemurmel und Getuschel, das jedes Mal kurz anschwoll, wenn jemand den Aufbahrungsraum betrat oder verließ.

Luca und Salvatore schlichen sich gemeinsam hinein. Leise drückten sie die Türe hinter sich ins Schloss, dann blieben sie eine Weile un- schlüssig stehen. Ihre Augen waren wie gebannt auf den in der Halbdunkelheit liegenden offenen Sarg gerichtet, aber der Leichnam selbst war von hier aus nicht zu sehen. Die Luft im Raum war stark verbraucht und ließ in Luca eine leichte Übelkeit aufkommen. Nachdem sich seine Augen an das schwache Licht gewöhnt hatten, schaute er sich um und konnte erkennen, dass die in Reihen aufgestellten Stühle größtenteils leer waren. Die vordersten drei Plätze waren besetzt, dann folgten zwei leere Sitzreihen, und zuhinterst saß auf den Plätzen ganz links und ganz rechts je ein Mann. Luca fühlte, wie sein Vater zu zittern begann. «Möchtest du noch einen Moment rausgehen?», flüsterte er ihm besorgt zu.

«Nein», erwiderte Salvatore kaum hörbar. «Bist du sicher? Wir können doch später ...»

Aber Salvatore hatte sich schon mit einem leichten Ruck von ihm gelöst und ging zögernd auf den Sarg zu. Luca folgte ihm.

Und nun sahen sie ihn daliegen, mit gespenstisch fahlem Antlitz, in einen schlichten schwarzen Anzug gekleidet, mit weißem Hemd, schwarzer Krawatte und schwarzen, im Kerzenlicht glänzenden Schuhen. Die von der jahrzehntelangen Arbeit klobig und rau gewordenen Hände waren über der Brust gefaltet und mit einem Rosenkranz umwickelt. Luca fühlte, dass sich in seinem Hals ein Kloß bemerkbar machte, der sich trotz mehrerer Versuche nicht hinunterschlucken ließ. Salvatore hielt derweil mit beiden Händen den Rand des Sarges umklammert, so krampfhaft, dass die Knöchel weiß hervortraten. Unvermittelt begann sich sein über den Sarg gebeugter Oberkörper zu schütteln, begleitet von einem unterdrückten Schluchzen. Luca stiegen bei dem erbärmlichen Anblick seines Vaters ebenfalls Tränen in die Augen. Er fühlte sich hilflos, wollte dem alten Mann tröstend die Hand auf die Schulter legen, doch dieser zuckte von ihm weg.

«Lass mich einen Moment allein», bat Salvatore mit brüchiger Stimme, ohne aufzublicken.

«Natürlich ... ich ... ich setze mich dort hinten hin ... nimm dir Zeit ... ich warte.»

Luca trat vom Sarg zurück und setzte sich auf einen der hinteren Stühle, von wo er ohnmächtig zuschauen musste, wie sein Vater litt. Er hatte Angst, dass der alte Mann zusammenbrechen würde, aber nach einigen Minuten schien sich dieser langsam zu beruhigen. Salvatore hatte aufgehört zu schluchzen, richtete sich nun wieder auf und holte ein Taschentuch hervor. Von hinten beobachtete Luca, wie er sich die Augen trocknete und anschließend kräftig schnäuzte. Umständlich steckte er das Taschentuch ein, griff erneut an den Rand des Sarges und schickte sich an, Carlo auf die Stirn zu küssen. 